\title{
Performance Analysis of Queuing Priority Schemes in cellular communication
}

\author{
Sayawu Yakubu Diaba ${ }^{1}$, Emmanuel Affume $^{2}$, Alewo Michea Oyibo ${ }^{3}$ \\ MSc Student KNUST, Kumasi, Ghana ${ }^{1}$ \\ Department of Electrical/Electronic Engineering, KNUST, Kumasi, Ghana ${ }^{2}$ \\ Lagos, Nigeria ${ }^{3}$
}

\begin{abstract}
In cellular networks, there exist fixed and dedicated channel for the entire duration of a set up call. The signal strength and interference at various locations differs within a cell and this affects the quality of the on-going call including handoff. This may require the transfer of the on-going call to another cell with a better signal strength. Given a fixed channel at a cell site, and assuming the arrivals of originating calls and handoff requests to be Poissonian, either process could result in queuing. This paper by using Matlab seeks to evaluate in terms of probability of blocking, which queuing system is more suitable when either or both priority are given and when no priority is given. It is observed that, for cell sites with traffic intensity to channel ratio of $0-0.75$ which is considered as not congested, a system of either queuing the originating or handoff calls can be employed. For a congested system in which the traffic intensity to channel ratio is $0.76-1$ there should be separate queuing of both the originating calls and the handoff calls. When the cell site is very congested that is, having a traffic intensity to channel ratio of greater than 1 , queuing of handoff calls provides the best network optimization.
\end{abstract}

Keywords: Signal strength, Originating calls, Handoff calls, Poissonian, and Queuing

\section{INTRODUCTION}

The need to increase capacity and reduce spectral assigned to the mother BS are used up. To mitigate the congestion in GSM called for a high power transmitter or bottleneck of force termination of an ongoing call and the large cell to be replaced with multiple low power blockage of destination call, several schemes had been transmitters or small cells each covering smaller service proposed.

area [1]. Each small cell is allocated a portion of the total The authors of [6] mentioned guard channels and queuing number of channels available to the entire system. To curb of handoff calls as a means to decrease the probability of interference, neighbouring cells are assigned different force termination while increasing the probability of call frequency channels. With a fixed frequency spectrum and the need for higher capacity, various frequencies are reused so long as interference is kept below the required levels. This is achieved with a minimum spacing of cells with the same frequency spectrum. Received Signal Strength (RSS) varies with location in a cell area. The further the Mobile Station (MS) is from the Base Station (BS), the weaker the RSS. In transition from one BS to another the RSS of the former decreases as that of the latter increases. This affects the quality of a setup call hence, the need for transfer of service to another BS. When an MS requests a connection, the BS sends the request to the Mobile Switching Centre (MSC) for a channel allocation. The MSC only allocates a channel when that channel is not already in use. This becomes a dedicated channel for the entire period of usage. When the MS strays beyond a certain range from the BS the RSS drops beyond a required level, there is the need for a transfer of service (channel) from one BS to another. This process of transferring an ongoing call from BS to another is called Call Handoff or Call Handover [2], [3], [4]. When the assigned channels of the receiving BS are occupied the call is forced to terminate. From the subscribers perspective it highly undesirable to terminate an ongoing call than to block an originating call [12], [16]. Likewise, originating calls are also blocked when the channel 


\section{SySTEM MODEL}

Consider a cell site in any of the operator that serves a number of people. For each BS, there exist a fixed number of voice channels, $\mathrm{N}$. These channels are apportioned to a subscriber on demand basis. However, these same channels are used to serve handoff or handover calls, which are also given on demand. When a subscriber requests service, a channel is allocated and remains dedicated for the entire duration (Holding time) of the call, $\mathrm{H}$. The service rate, $\mu$, which is the frequency of the allocation of $\mathrm{N}$ to a subscriber, is the reciprocal of $\mathrm{H}$. Therefore, the average calling time or holding time per subscriber is given by $\mathrm{H}=1 / \mu$.

Consider similar subscribers requesting for N. These requests could be from originating calls or a handoff requests. The frequency at which these requests arrive at the MSC is known as call arrival rate, $\lambda$. For originating calls it is denoted by $\lambda_{1}$ and $\lambda_{2}$ for handoff calls. When these arrivals are in excess of the total number of channels available, a method of queuing can be employed. Where, $M_{1}$ refers to the size of queue for originating calls and $M_{2}$ refers to the size of queue for handoff calls. Therefore, at a particular cell site, the total traffic intensity due to originating calls and handoff call is given by: $a=\left(\left(\lambda_{1}+\lambda_{2}\right)\right) / \mu$ As a result, the traffic intensity due originating call is given by: $b_{1}=\lambda_{1} / \mu$. The traffic intensity due handoff calls is also given by: $b_{2}=\lambda_{2} / \mu$

\section{A. Call Blocking Probability}

Competition for channels is the main reason for call blocking. When requests at MSC exceed the available channels at a particular cell site, any excess requests are blocked in order to service the already established ones. The call blocking probability (CBP) is defined as the probability that the new calls finds all the channels busy and blocked [13]. In this paper, blocking of originating calls and dropping of handoff calls requests are considered together. Blocking is determined by a dimensionless unit known as Erlang. It is the measure of carried load on service-providing elements such as telephone circuits or telephone switching equipment. It is also the measure of the Grade of Service (GOS) for a trunked system that provides no queuing for blocked calls. Erlang B is based on the assumptions:

* Call requests are memory less, implying that all users, including blocked users, may request a channel at any time.

* $\quad$ All free channels are fully available for servicing calls until all channels are occupied.

* The probability of a user occupying a channel (called the service time) is exponentially distributed. Longer calls are less likely to happen as described by an exponential distribution.
* There are a finite number of channels available in the trunking pool.

* Traffic requests are described by a Poisson distribution which implies exponentially distributed call inter-arrival times.

* Inter-arrival times of call requests are independent of each other.

* The number of busy channels is equal to the number of busy users, and the probability of blocking is given as:

$P_{b}=\frac{\frac{A^{c}}{\mathrm{C} !}}{\sum_{k=0}^{c} \frac{A^{k}}{k !}}$

$\mathrm{C}$ is the number of channels, a, is the offered traffic.

Equation (1) is the probability of blocking when there is no any queue. An extension to equation (1) is the instance where excess call requests are not blocked but rather queued with the assumptions that:

* Callers never ring off whilst in queue.

* All calls start and end in the same time period being estimated for.

* Callers never try to call back after having hanged up while in queue.

$P_{b q}(0)=\left[C ! \sum_{C=0}^{C-1} \frac{A^{c-C}}{\mathrm{c} !}+\frac{1-\left(\frac{b_{1}}{C}\right)^{M_{1}+1}}{1-\left(\frac{b_{1}}{C}\right)}\right]^{-1}$

\section{Case 2}

When only the originating calls but not the handoff calls are queued, the blocking probability for originating calls is given by:

$$
B_{o q}=\left(\frac{b_{1}}{C}\right)^{M_{1}} P_{b q}(0)
$$

The resulting blocking probability for handoff calls is given by:

$B_{h q}=\frac{1-\left(\frac{b_{1}}{C}\right)^{M_{1}+1}}{1-\left(\frac{b_{1}}{C}\right)} \quad P_{b q}(0)$

\section{Case 3}

When the handoff calls are queued but not the originating calls, the blocking probability for handoff calls is presented as: 


$$
B_{h q}=\left(\frac{b_{2}}{C}\right)^{M_{2}} P_{b q}(0)
$$

And the blocking probability for origination calls is:

$$
B_{o q}=\frac{1-\left(\frac{b_{2}}{C}\right)^{M_{2}+1}}{1-\left(\frac{b_{2}}{C}\right)} P_{b q}(0)
$$

\section{Simulation MODEL}

Dynamic system level simulations are performed to examine the impacts of queuing of originating call and handoff calls on mobile communication networks.

The following parameters are used for the simulation in respect to Valley View DIA cell site:

- $\quad$ Originating call arrival rate: 0.0172 per sec.

- $\quad$ Handoff call arrival time: 0.08 per sec.

- $\quad$ Average holding time: $49.85 \mathrm{sec}$.

- $\quad$ Number of channels: 13

- $\quad$ Traffic intensity: 102.67 Erlang.

- Initial probability of delay:0.018

However, the parameters used for simulation at the Achimota DIA cell site are totally different and presented below:

- $\quad$ Originating call arrival rate: 4.2030 per seconds.

- Handoff arrival rate of: 0.5018 per seconds.

- $\quad$ Number of channels: 114

- $\quad$ Mean holding time: 21.57

- Traffic intensity: 101.48 Erlang.

Finally, the below parameters are used for Adenta DIA

- $\quad$ Originating call arrival rate: 6.8102 per seconds.

- $\quad$ Handoff arrival rate of: 1.0141 per seconds.

- $\quad$ Number of channels: 98

- $\quad$ Mean holding time: 12.17

- Traffic intensity: 95.22 Erlang.

\section{Simulation Results}

Data were obtained from a leading telecom operator, Mat lab is used to analyse the two scenarios, namely, queuing the originating calls and queuing the handoff calls. It is observed that, both scenarios have different impact on the probability of blocking on either one.

\section{A. Result for Valley View (DIA)}

From the Figure 4.1, it is deduced that the blocking probability decrease as the queue size increases, yet, it does not conform to the general concept of blocking probability.

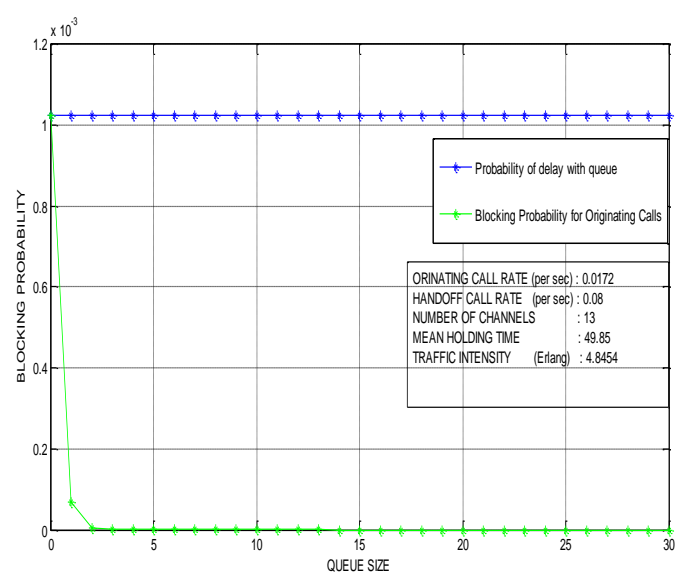

Fig 4.1: Queuing of OC Blocking Probability of OC (Valley View-DIA)

Blocking probability of handoff calls when originating calls are queued is explored next. The parameters used in analyzing the blocking probability of originating calls when originating calls are queued are same used here. From Figure 4.2, it is observed that, queuing originating calls have an effect on the blocking probability of handoff calls; this effect is insignificant since it does not really make any difference due to the initial probability of blocking.

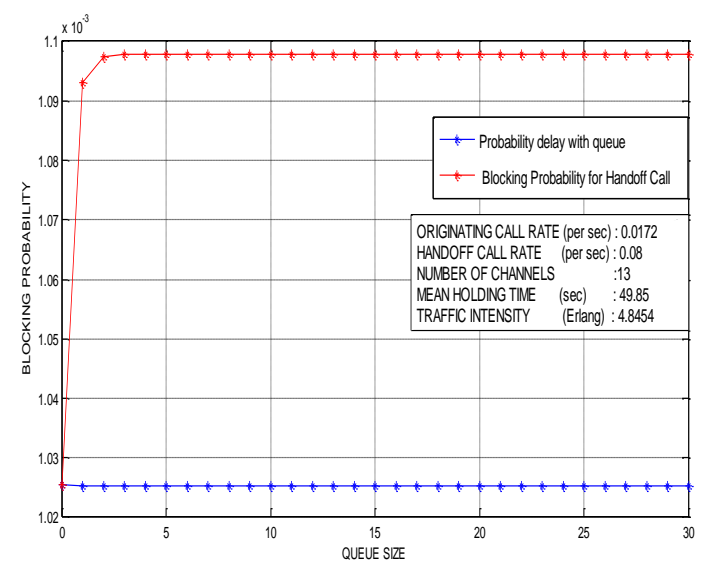

Figure 4.2: Queuing of OC Blocking Probability of HC (Valley View-DIA)

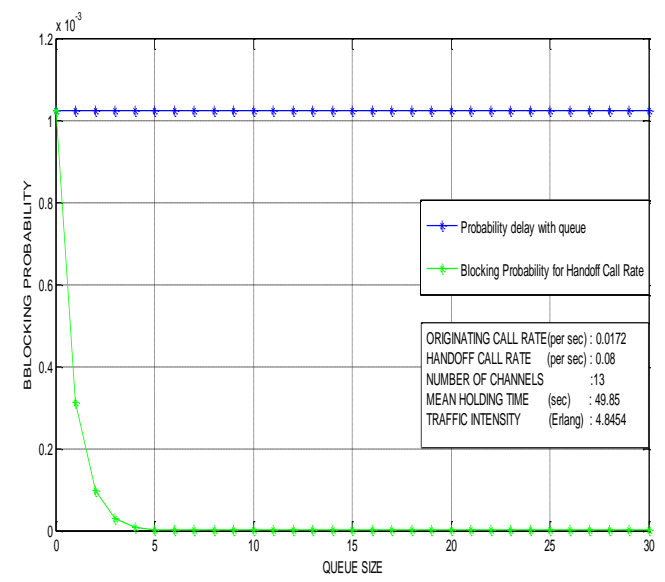

Figure 4.3: Queuing of HC Blocking Probability of HC (Valley View-DIA) 


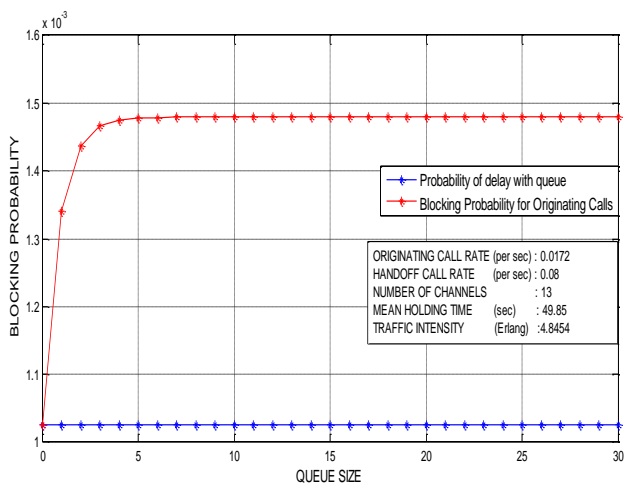

Fig 4.4: Queuing of HC Blocking Probability of OC (Valley View-DIA)

B. Result for Achimota (DIA)

The traffic intensity generated from the above stated data is 101.48 Erlang while the blocking probability at 0 queue size is 0.02 . From the figure, it is deduced that blocking probability decrease as the queue size increases; it reduced from the initial probability of blocking of 0.02 at 0 queue size to 0 at 22 queue size, a gradual reduction though, but does conform to the general concept of blocking probability as in the [13].

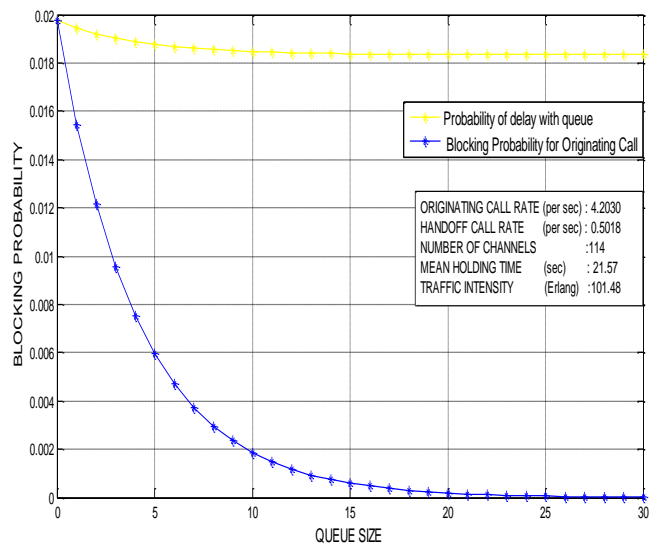

Figure 4.9: Queuing of OC blocking probability of OC (Achimota- DIA)

Since originating calls are given priority, there exists no room to accommodate handoff requests. This implies that any handoff request at Achimota cell site is dropped as soon as it comes. These results in the increase in blocking probability for handoff calls from a little above 0.02 to almost 0.09 as the queue sizes for the originating calls increased.

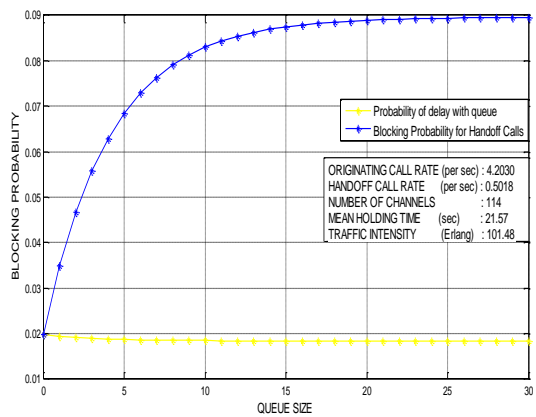

Figure 4.10: Queuing of OC blocking probability of HC (Achimota DIA)

Copyright to IJARCCE

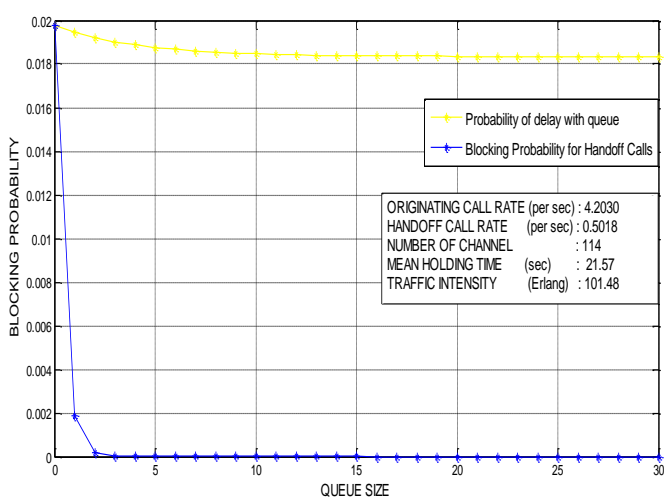

Fig 4.11: Queuing of $\mathrm{HC}$ blocking probability of HC (Achimota - DIA)

It is observed from the plot that blocking probability of handoff calls drops sharply to 0 just at queue size of 3 . This is due to the fact that, there are small number of handoff calls and a relatively large number of channels and therefore, the handoff calls get the channels as soon as the requests are put in.

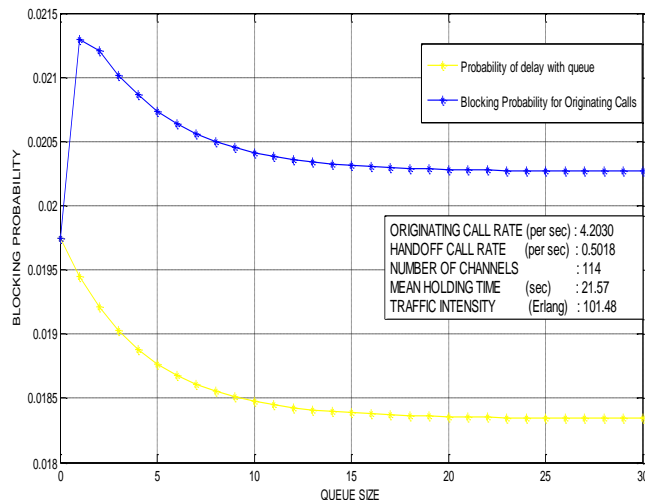

Fig 4.12: Queuing of HC blocking probability of OC (Achimota - DIA)

C. Result for Adenta (DIA)

From the figure, it is deduced that blocking probability decrease as the queue size increases; it reduced from the initial probability of blocking of 0.02 at 0 queue size to 0 at 22 queue size, a gradual reduction though, but does conform to the general concept of blocking probability as in the [].

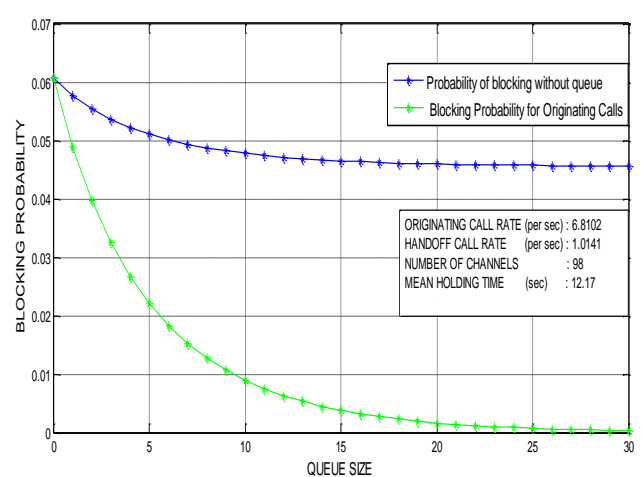

Fig 4.13: Queuing of OC blocking probability of OC (Adenta - DIA) 


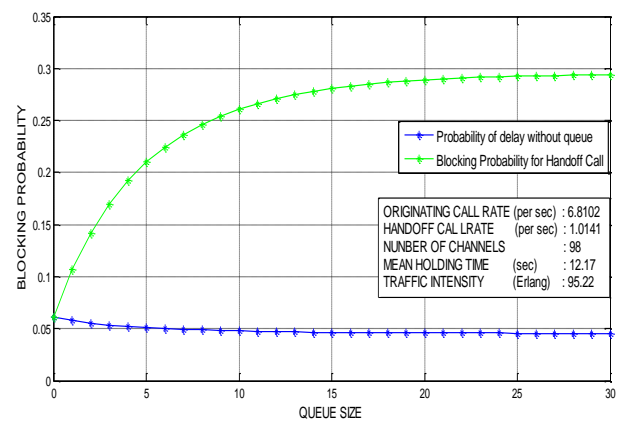

Fig 4.14: Queuing OC blocking probability of HC (Adenta -DIA)

This very high probability of blocking occurs due to the high originating calls arrival rate. Since the originating calls rate is very high, all the available channels are used by it and the handoff calls are dropped once the available channels are used up.

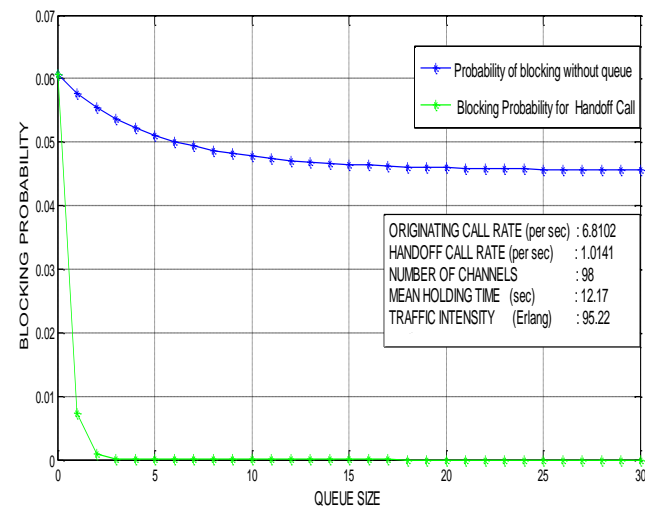

Fig 4.15: Queuing HC blocking probability of HC (Adenta -DIA)

It is deduced from the graph that, the blocking probability of handoff calls dropped massively from 0.06 at 0 queue size to 0 at just 3 queue size. This means at Adenta cell site a total queue size of 3 is enough to perfectly handle handoff calls when handoff calls are queued.

Next, we consider the impact queuing handoff calls has on originating calls.

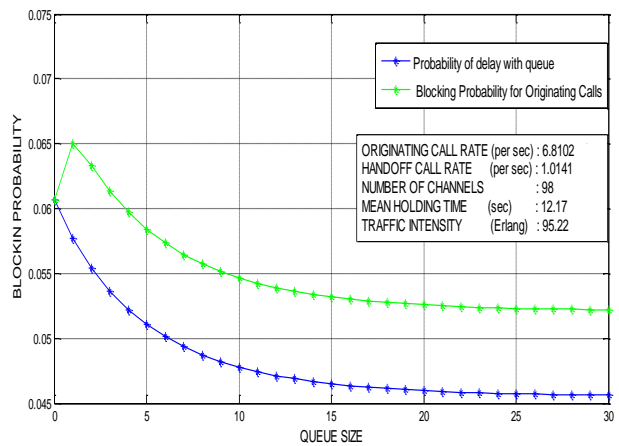

Fig 4.16: Queuing HC blocking probability of OC (Adenta -DIA)

\section{CONClusion}

At Valley View cell site the traffic intensity per channel value is 0.373 , and hence, the simulation results conformed to the case in the reference. It is observed from the graphs that, queuing originating calls caused an increase in the handoff blocking probability and queuing of handoff calls also caused an increase in originating calls, yet, this blocking actually was also within the desired blocking limit of 0.02 . However, for such cell sites, either queue performs well.

At Achimota, there was a traffic intensity to channel rate of 0.89 with an initial probability of blocking of 0.02 . The simulation results conformed to the reference model. Queuing originating calls results in a decrease in blocking probability of originating calls but queuing originating calls almost caused a total blockage of handoff calls. On the other hand, when handoff calls are queued, the blocking probability of handoff calls drop shapely to 0 ensuring blocking free system for the handoff calls. The blocking probability of originating calls increased initially and then reduced, however, the reduction was still above the grade of service $(\mathrm{GoS})$. Such a cell site will perform better when different queues are employed for the originating calls and handoff calls.

From the Adenta cell site results, we noticed a significant decrement in the blocking probability of the originating calls when the originating calls are given priority but the blocking probability of handoff calls increased to 0.24 which is above the GoS. When the handoff calls are queued the blocking probability of the handoff calls again dropped to 0 at a very small queue size of 3 , making the system convenient for the handoff calls. The impact of originating calls at Adenta cell site is the same as that of Achimota. Also, the reduction is above the GoS. Hence, there is the need to implement different queue for the originating calls and the handoff calls respectively.

\section{REFERENCES}

11] K. S. Trivedi, S. Dharmaraja and M Xiaomin. " Analytic modeling of handoffs in wireless cellular networks" CACC Department of ECE, Duke University, Durham, NC 27708, USA.

[2] N. D. Tripathi, N. J. H. Reed and H. F. VanLandingham. "Handoff in Cellular Systems". Virginia Tech.KS

[3] S. Dharmaraja, K.S. Trivedi and D. Logothetis. "Performance modeling of wireless networks with generally distributed handoff interarrival times". CACC, Department of ECE, Duke University, Durham, NC 27708, USA.

[4] D Kaurland N. Kumar "Performance Analysis of Handoff in CDMA Cellular System". International Journal of Computers \& Technology. ISSN 22773061, July 20, 2013.

[5] N. Ekiz, T. Salih, S. Kucukoner and K. Fidanboylu. "An Overview of Handoff Techniques in Cellular Networks" International Journal of Information and Communication Engineering 2:6 2006.

[6] S. S. Moghaddam and A. H. Khodadadi. "A Novel Measurement Based Channel Assignment Method" (New Priority Method Based on Measurement of Received Power). International Symposium on Telecommunications. September 10-12, 2005 Shiraz, Iran

[7] H. H. Nour el, M. Ouacifi, R. Bouchouareb, M. Ourghi, M. Gareh and D. Benatia. "The Handover in the Constellations of Satellites in Low Orbit". International Journal of Advanced Science and Technology. Vol. 41, April, 2012

[8] S. Louvros, J. Pylarinos and S. Kotsopoulos. "Handoff multiple queue model in microcellular networks" Computer Communications 30 (2007) 396-403.

[9] A.Z. Melikov and A.T. Babayev. "Refined approximations for performance analysis and optimization of queueing model with guard channels for handovers in cellular networks". Computer Communications 29 (2006) 1386-1392.

[10] B. Awasthi. "Hand Off Problem Analysis In Personal Communication System Using Splitted-Rating Channel Technique". International Journal of Scientific \& Technology Research Volume 3, Issue 1, January 2014. ISSN 2277-8616

[11] T. Salih, K. Fidanboylu "Modeling and analysis of queuing handoff calls in single and two-tier cellular networks" Computer Engineering Department, Fatih University, 34500 Istanbul, Turkey.

[12] L. Chow-Sing and H. Pin-Jing. "Dynamic Handoff Priority Adjustment Based on Mobility-Awareness in Multimedia Cellular Networks" Int. J. Communications, Network and System Sciences, 2011, 4, 495-506.

[13] W.C.Y. Lee. "Wireless and Cellular Telecommunications" Third Edition. 